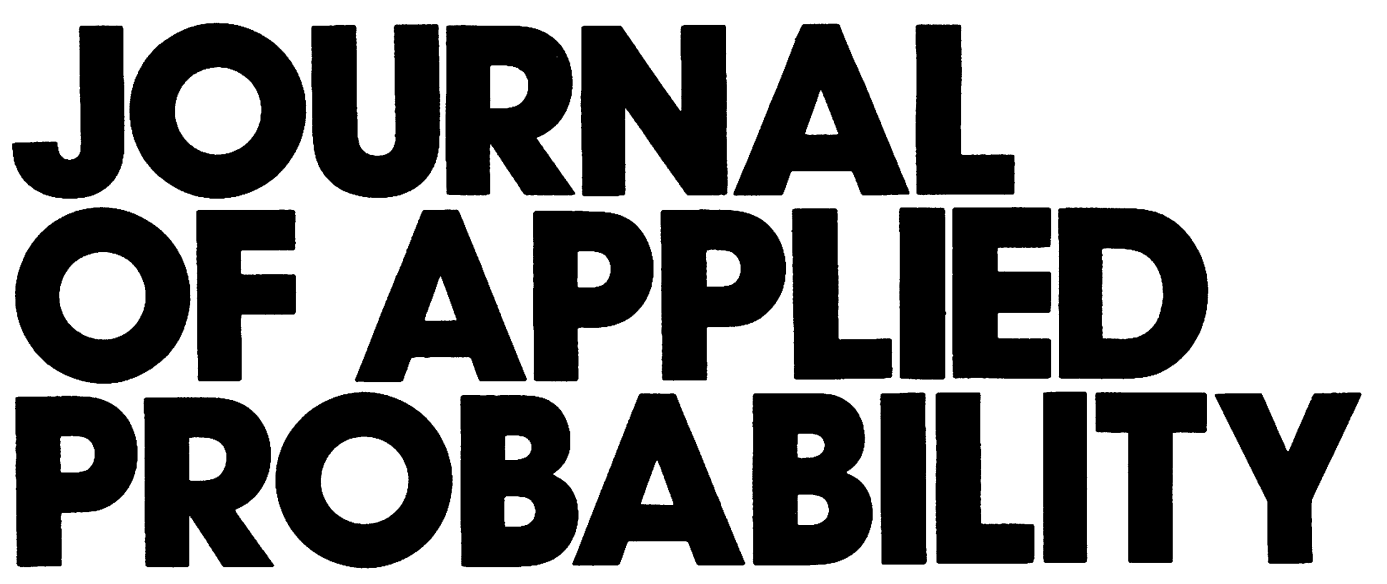

VOLUME $23 \quad 1986$

AT Editor-In-CHIEF J.GANI 


\section{Volume 23 Number 2}

\section{Research Papers}

265 ANDERS MARTIN-LÖF. Symmetric sampling procedures, general epidemic processes and their threshold limit theorems

283 PETER DONNELLY. A genealogical approach to variable-population-size models in population genetics

297 REINHARD HÖPFNER. Some results on population-size-dependent Galton-Watson processes

307 A. M. KELLERER. The variance of a Poisson process of domains

322 JÜRG HÜSLER. On point processes on the circle

332 CHERN-CHING CHAO AND JOHN SLIVKA. Some exact distributions of a last one-sided exit time in the simple random walk

341 G. MAZZIOTTO. Approximations of the optimal stopping problem in partial observation

355 PAUL DEHEUVELS AND JOSEF STEINEBACH. Exact convergence rate of an Erdös-Rényi strong law for moving quantiles

370 P. L. DAVIES. Rates of convergence to the stationary distribution for $k$-dimensional diffusion processes

385 ENZO ORSINGHER. A planar random motion governed by the two-dimensional telegraph equation

398 V. GIORNO, A. G. NOBILE, L. M. RICCIARDI AND L. SACERDOTE. Some remarks on the Rayleigh process

409 A. THAVANESWAREN AND M. E. THOMPSON. Optimal estimation for semimartingales

418 DENNIS V. LINDLEY AND NOZER D. SINGPURWALLA. Multivariate distributions for the life lengths of components of a system sharing a common environment

432 TERJE AVEN AND BO BERGMAN. Optimal replacement times - a general set-up

443 MASAKIYO MIYAZAWA. Approximations of the queue-length distribution of an $M / G I / s$ queue by the basic equations

459 V. V. KALASHNIKOV AND S. T. RACHEV. Characterizations of inverse problems in queueing and their stability

$474 \mathrm{~J}$. GEORGE SHANTHIKUMAR AND DAVID D. YAO. The effect of increasing service rates in a closed queueing network

484 M. H. VAN HOORN AND L. P. SEELEN. Approximations for the $G I / G / c$ queue

495. BRUNO VISCOLANI. An adaptive multistage queueing system

\section{Short Communications}

504 N. C. WEBER. The Wright-Fisher model with varying selection

509 KIM-ANH DO AND HERBERT SOLOMON. A simulation study of Sylvester's problem in three dimensions

514 DROR ZUCKERMAN. Optimal stopping in a continuous search model

519 KYLE SIEGRIST. Markov chains with binomial time change

$524 \mathrm{~J}$. MICHAEL STEELE AND LUKE TIERNEY. Boundary domination and the distribution of the largest nearest-neighbor link in higher dimensions

529 R. J. MARTIN. A note on the asymptotic eigenvalues and eigenvectors of the dispersion matrix of a second-order stationary process on a $d$-dimensional lattice

536 TOSHIO NAKAGAWA. Periodic and sequential preventive maintenance policies

543 WILLIAM A. MASSEY. A family of bounds for the transient behavior of a Jackson network

550 ROBERT B. COOPER AND SHUN-CHEN NIU. Beneš's formula for $M / G / 1$-FIFO 'explained' by preemptive-resume LIFO

$555 \mathrm{R}$. SZEKLI. On the concavity of the waiting-time distribution in some $G I / G / 1$ queues

562 Correction

Published by the Applied Probability Trust in association with the London Mathematical Society (C) 1986 


\title{
THE VARIANCE OF A POISSON PROCESS OF DOMAINS
}

\author{
A. M. KELLERER, ${ }^{*}$ University of Würzburg
}

\begin{abstract}
A familiar relation links the densities that result for the intersection of a convex body and straight lines under uniform isotropic randomness with those that result under weighted randomness. An extension of this relation to the intersection of more general domains is utilized to obtain the variance of the $n$-dimensional measure of the intersection of two bodies under uniform isotropic randomness. The formula for the variance contains the point-pairdistance distributions for the two domains - or the closely related geometric reduction factors. The result is applied to derive the variance of the intersection of a Boolean scheme, i.e. a stationary, isotropic Poisson process of domains, with a fixed sampling region.

RANDOM INTERSECTION OF GEOMETRIC OBJECTS; VARIANCE OF OVERLAP; LATTICE PROBLEM; POISSON PROCESS; BOOLEAN SCHEME; POINT-PAIR-DISTANCE DISTRIBUTION
\end{abstract}

\section{Introduction}

The fundamental formula of Blaschke [2] and Santaló [25] determines, for isotropic uniform randomness, the average Minkowski functionals of the intersection of two domains, $F_{0}$ and $F_{1}$. The equation contains only the Minkowski functionals of $F_{0}$ and $F_{1}$ [2], [25]. The formula can be extended [5], [26] to the average of the Minkowski functionals of a Boolean scheme [16], [17] of geometric objects, i.e. a stationary Poisson process of independent geometric objects. The relations have been given also for the intersection of a Boolean scheme with a fixed sampling domain [9], [11]. No relations of comparable simplicity exist for the variance of the measures of the intersection. However, Robbins derived a general form of the integrals for the moments of the measure of random sets [21], and he [22], Bronowski and Neyman [3], Santaló [24], and Garwood [7] used his result to derive the variances of the overlap of circles and rectangles. Miles [18] has obtained formulae for the variance of the intercept of

Received 7 March 1985; revision received 18 April 1985.

* Postal address: Institut für Med. Strahlenkunde der Universität Würzburg, Versbacher Str. 5, D-8700 Würzburg, W. Germany.

Work supported by Euratom Contract BI-6-0013 D(B) and Contract 96731 with GSI (Gesellschaft für Schwerionenforschung, Darmstadt). 
convex bodies by linear subspaces. Pitts [20] has recently treated a problem closely related to the random intersection of geometric objects, and he has given approximation formulze for the variance of the Poisson process.

In the following an equation will be derived that is more specific than Robbins' theorem. It expresses the variance of the measure of the intersection of two domains in terms of the point-pair-distance distributions for the two domains. The result is then applied to the intersection of a Boolean scheme with a sampling domain, i.e. a finite field of observation. The treatment utilizes a relation between densities for uniform isotropic randomness and weighted randomness of the intersection of two domains. This relation has been obtained by Kingman [15] for the intercept of straight lines and a convex body. It is here extended to the intercept of two piecewise smoothly bounded domains.

\section{Isotropic uniform randomness and weighted randomness}

The subsequent derivation parallels the one that Coleman [4] has utilized to demonstrate the relation between isotropic uniform randomness and weighted randomness of straight lines intercepting a domain. Two piecewise smoothly bounded domains, $F_{0}$ and $F_{1}$, in $R^{n}$ are considered. Each of the domains has a reference point. $F_{0}$ is immobile with its reference point on the origin of a Cartesian coordinate system. $F_{1}$ is randomly placed; its position is determined by the coordinates, $\boldsymbol{x}$, of its reference point and by a parameter vector, $\boldsymbol{u}$, which determines the orientation. The measures of $F_{0}$ and $F_{1}$ are $A_{0}$ and $A_{1}$. Here and below the term measure is used for the $n$-dimensional measure.

Density for isotropic uniform randomness. Isotropic uniform randomness ( $\mu$-randomness) is obtained by positioning $F_{1}$ randomly oriented with its reference point randomly placed in a sufficiently large domain containing $F_{0}$. Eliminating all positions with $F_{0} \cap F_{1}=0$ one has the density

$$
p_{\mu}(\boldsymbol{x}, \boldsymbol{u})= \begin{cases}p_{\mu}(\boldsymbol{x} \mid \boldsymbol{u}) \cdot p_{\mu}(\boldsymbol{u}) & \text { for }(\boldsymbol{x}, \boldsymbol{u}) \mid F_{0} \cap F_{1} \neq 0 \\ 0 & \text { otherwise. }\end{cases}
$$

The non-zero part of the density is the product of

$$
p_{\mu}(x \mid u)=\frac{1}{\gamma(u)}
$$

and

$$
p_{\mu}(u)=\frac{\gamma(u) h(u)}{\gamma},
$$

$h(u)$ is the density that corresponds to isotropic random orientation, $\gamma(u)$ is the measure of the Minkowski sum of $F_{0}$ and $F_{1}$ with inclination $u$, and $\gamma$ is the 
average of $\gamma(\boldsymbol{u})$ over all inclinations:

$$
\gamma=\int \gamma(u) h(u) d u
$$

Accordingly one has

$$
p_{\mu}(x, u)=\frac{h(u)}{\gamma} \text { for }(x, u) \mid F_{0} \cap F_{1} \neq 0 .
$$

Density for weighted randomness. Weighted randomness ( $\nu$-randomness) is obtaind by the selection of two independent random points, $\boldsymbol{x}_{0} \in F_{0}$ and $\boldsymbol{x}_{1} \in F_{1}$, and by positioning $F_{1}$ with random inclination, $u$, so that the two points are superimposed. $x_{0}$ and $x_{1}$ are the coordinates of the random points relative to the reference points of $F_{0}$ and $F_{1}$. The resulting position of $F_{1}$ is $(x, u)$ with $x=x_{0}-x_{1}$. The density is

$$
p_{\nu}\left(x_{0}, x_{1}, u\right)= \begin{cases}\frac{h(u)}{A_{0} A_{1}} & \text { for } x_{0} \in F_{0} \text { and } x_{1} \in F_{1} \\ 0 & \text { otherwise. }\end{cases}
$$

Let $a(x, u)$ be the measure of the intersection. The marginal density $p_{\nu}(x, u)$ is then

$$
\begin{aligned}
p_{\nu}(x, u) & =\frac{a(x, u) h(u)}{A_{0} A_{1}}=a(x, u) \cdot p_{\mu}(x, u) \frac{\gamma}{A_{0} A_{1}} \\
& =a(x, u) \cdot p_{\mu}(x, u) / E_{\mu} a .
\end{aligned}
$$

This is the $\mu$-density biased with the measure, $a(x, u)$, of the intersection $F_{0} \cap F_{1}$. The normalization condition

$$
E_{\mu} a=\frac{A_{0} A_{1}}{\gamma}
$$

is identical to the formula of Blaschke-Santalo for the average measure of $F_{0} \cap F_{1}$ under uniform randomness.

From Equation (7) one obtains the corresponding relation between the $\mu$-density and the $\nu$-density of the measure, $a$, of $F_{0} \cap F_{1}$ :

$$
p_{\nu}(a)=\frac{a}{E_{\mu} a} p_{\mu}(a)
$$

or, in the more general formulation that includes any singularities of the densities,

$$
d P_{\nu}(a)=\frac{a}{E_{\mu} a} d P_{\mu}(a)
$$

where $P_{\nu}(a)$ and $P_{\mu}(a)$ are the distribution functions. 
Equations (9) and (10) generalize the familiar relation for the intersection of straight random lines with convex bodies.

\section{Variance of the intersection of two domains}

From Equations (9) and (10) one obtains a relation for the second moment and the variance of the measure under $\mu$-randomness:

$$
E_{\mu} a^{2}=E_{\nu} a E_{\mu} a
$$

and

$$
\sigma_{\mu}^{2}=\left(E_{\nu} a-E_{\mu} a\right) E_{\mu} a .
$$

$E_{\mu} a$ is known from Equation (8), and $E_{\nu} a$ will subsequently be expressed in terms of the point-pair-distance distributions of $F_{0}$ and $F_{1}$, or in terms of the closely linked geometric reduction factors.

Robbins' theorem is required only in its simplest, intuitively evident form. It is nevertheless informative to recall the theorem, as it indicates, even without further consideration, implicit connections to the present result.

Robbins' theorem. Consider a random measurable subset, $F$, of $R^{n}$ with measure $a(F)$. For any set of points $\left(x_{1}, x_{2}, \cdots, x_{k}\right)$ of $R^{n}$ let $p\left(x_{1}, x_{2}, \cdots, x_{k}\right)=$ $\operatorname{Pr}\left(x_{1} \in F\right.$, and $x_{2} \in F, \cdots$ and $\left.x_{k} \in F\right)$. Robbins [21] obtained the $k$ th moment of $a(F)$ as an integral over the product space $R^{k n}$ :

$$
E a(F)^{k}=\int_{R^{k n}} p\left(x_{1}, x_{2}, \cdots, x_{k}\right) d x_{1} d x_{2} \cdots d x_{k} .
$$

In actual cases it can be difficult to derive the function $p\left(x_{1}, x_{2}, \cdots, x_{k}\right)$. Solutions have, therefore, been obtained only for relatively simple cases involving spheres or aligned parallelepipeds [3], [21], [22], [24].

Point-pair-distance distributions and geometric-reduction factors. Let $P$ be a random point, uniformly distributed in $F$, and let $P^{\prime}$ be the translate of $P$ by distance $x$ in uniformly distributed random direction. The function $U(x)$ is then defined as the probability that $P^{\prime}$ is also a point of $F$ :

$$
U(x)=\operatorname{Pr}\left(P^{\prime} \in F \mid P \in F\right) .
$$

In applications to radiation dosimetry $U(x)$ has been termed the geometric reduction factor [1]. Enns and Ehlers [6] have utilized the same concept in considerations of chord-length distributions.

$U(x)$ is linked to the point-pair-distance distribution of $F$, i.e. to the density, $p(x)$, of distances, $x$, between pairs of random points distributed uniformly and independently in $F$. If $w_{n}$ is the surface of the unit ball in $R^{n}$ and $A$ is the measure (length, area, volume in $R^{1}, R^{2}, R^{3}$ ) of $F$ : 


$$
p(x)=U(x) w_{n} x^{n-1} / A .
$$

Equation (15) holds because - in accordance with Robbins' theorem for $k=1$ - the term $U(x) w_{n} x^{n-1} d x$ is the expected measure of $F$ at distance $x$ to $x+d x$ from a random point in $F$. Geometric reduction factors or point-pair-distance distributions are known for a variety of simple geometric configurations, such as cylinders, spheroids, ellipses, or rectangles (see appendix). For more complicated shapes they may require numerical integrations or Monte Carlo computations.

Formula for the variance. According to the definition of weighted randomness two random points of $F_{0}$ and $F_{1}$ are superimposed. $U_{0}(x)$ and $U_{1}(x)$ are the probabilities that the translate, $S^{\prime}$, by distance $x$ in random direction of the point, $S$, of superposition leads to a point in $F_{0}$ and $F_{1}$, respectively. Due to the random orientation of $F$. relative to $F_{0}$ the two probabilities are uncorrelated; $U_{0}(x) U_{1}(x)$ is therefore the probability of $S^{\prime}$ being contained in $F_{0} \cap F_{1}$. Accordingly, and again with Robbins' theorem for $k=1$, the expected measure of $F_{0} \cap F_{1}$ is

$$
E_{\nu} a=\int_{0}^{d} w_{n} x^{n-1} U_{0}(x) U_{1}(x) d x=A_{0} \int_{0}^{a} p_{0}(x) U_{1}(x) d x
$$

$d=\min \left(d_{0}, d_{1}\right)$, with $d_{0}$ and $d_{1}$ being the diameters, i.e. maximal point-pair distances, of $F_{0}$ and $F_{1}$.

Combining Equations (12) and (16) one obtains, either in terms of the geometric reduction factors or the point-pair-distance distributions, the relation for the variance of the measure of the intersection of two domains under uniform, isotropic randomness in $R^{n}$ :

$$
\begin{aligned}
\sigma^{2} & =\left(\int_{0}^{d} w_{n} x^{n-1} U_{0}(x) U_{1}(x) d x-E_{\mu} a\right) E_{\mu} a \\
& =\left(A_{0} \int_{0}^{d} p_{0}(x) U_{1}(x) d x-E_{\mu} a\right) E_{\mu} a
\end{aligned}
$$

with $w_{1}=2, w_{2}=2 \pi, w_{3}=4 \pi$.

As stated in Equation (8), one has $E_{\mu} a=A_{0} A_{1} / \gamma$. For convex regions, $F_{0}$ and $F_{1}$, one can give the explicit relations. In $R^{1}$, with $A_{0}$ and $A_{1}$ being the lengths of $F_{0}$ and $F_{1}$,

$$
\gamma=A_{0}+A_{1} .
$$

In $R^{2}$, with $A_{0}$ and $A_{1}$ the areas, and $S_{0}$ and $S_{1}$ the perimeters of $F_{0}$ and $F_{1}$,

$$
\gamma=A_{0}+S_{0} S_{1} / 2 \pi+A_{1} .
$$

In $R^{3}$, with $A_{0}$ and $A_{1}$ the volumes, $S_{0}$ and $S_{1}$ the surface areas, and $M_{0}$ and $M_{1}$ 
the integral mean curvatures of $F_{0}$ and $F_{1}$ :

$$
\gamma=A_{0}+\left(S_{0} M_{1}+S_{1} M_{0}\right) / 4 \pi+A_{1} .
$$

\section{Extension of the result to the Poisson process}

Geometric reduction factor for the realization of a Boolean scheme. A Boolean scheme in $R^{n}$ is generated by the domains $C(p)$. Their shape is taken to be sufficiently regular and to depend on the parameter vector $p$. Each domain has a reference point, termed the centre, and is randomly oriented. The positions of the centres and the inclinations are uniformly, isotropically and independently distributed. The density of the Poisson intensity in the parameter space is $\lambda(p)$, i.e., the expected number of centres per unit volume of $R^{n}$ is $\lambda(p) d p$ for domains corresponding to the element $d p$ of the parameter space. $B$ is the union of all domains that form the Poisson process, and the geometric reduction factor of $B$ is denoted by $U_{B}(x)$ :

$$
U_{B}(x)=\operatorname{Pr}\left(P^{\prime} \in B \mid P \in B\right)
$$

where $P$ is a point uniformly distributed in $R^{n}$, and $P^{\prime}$ is its translate by distance $x$ in random direction.

The expected number of domains that cover - i.e., contain - the point $P$ is $\Psi=\int A(p) \lambda(p) d p$, where $A(p)$ is the measure of $C(p)$. The quantity $\Psi$ is termed nominal coverage. Due to the independence of the domains in the Poisson process, one has the probabilities for $P$ being covered or uncovered:

$$
p=\operatorname{Pr}(P \in B)=1-\exp (-\Psi) \text { and } q=\operatorname{Pr}(P \in \bar{B})=\exp (-\Psi) .
$$

With

$$
\operatorname{Pr}\left(P^{\prime} \in B\right)=p \cdot \operatorname{Pr}\left(P^{\prime} \in B \mid P \in B\right)+q \cdot \operatorname{Pr}\left(P^{\prime} \in B \mid P \in \bar{B}\right)
$$

and with the abbreviation

$$
T_{B}(x)=\operatorname{Pr}\left(P^{\prime} \in \bar{B} \mid P \in \bar{B}\right)
$$

one obtains

$$
p=p U_{B}(x)+q\left(1-T_{B}(x)\right)
$$

or

$$
U_{B}(x)=1-\left(1-T_{B}(x)\right) /(\exp (\Psi)-1) .
$$

An additional consideration provides the function $T_{B}(x)$. Let $\nu=\nu_{1}+\nu_{2}$ be the number of domains that cover $P^{\prime}$, where $\nu_{2}$ is the number of domains that cover both $P$ and $P^{\prime}$, while $\nu_{1}$ is the number of domains that cover $P^{\prime}$ but not $P$. The expectation of $\nu_{2}$ is 


$$
E \nu_{2}=\int A(p) \lambda(p) U(x, p) d p=U(x) \Psi
$$

where $U(x, p)$ is the geometric reduction factor of the domain $C(p)$, and $U(x)$ is the weighted average of the geometric reduction factors for all domains:

$$
U(x)=\int A(p) \lambda(p) U(x, p) d p / \int A(p) \lambda(p) d p .
$$

With $E \nu=\Psi$ one obtains

$$
E \nu_{1}=E \nu-E \nu_{2}=(1-U(x)) \Psi .
$$

Due, again, to the independence of the domains in the Poisson process one has

$$
T_{B}(x)=\operatorname{Pr}\left(\nu_{1}=0 \mid P \in \bar{B}\right)=\operatorname{Pr}\left(\nu_{1}=0\right)=\exp (-(1-U(x)) \Psi) .
$$

The geometric reduction factor for the configuration, $B$, formed by the Poisson process is, therefore,

$$
U_{B}(x)=1-\exp (-\Psi)+\exp (-\Psi)(\exp (\Psi U(x))-1) /(\exp (\Psi)-1) .
$$

The last term vanishes for distances, $x$, exceeding the maximum diameter of the domains $C(p)$.

Variance of the intersection with a sampling domain. In practical applications one registers the configuration, $I=B \cap W$, formed by the Poisson process in a window, $W$, that serves as sampling domain. Let $A_{\mathrm{w}}$ be the measure of $W$, and $p_{\mathrm{w}}(x)$ and $U_{\mathrm{w}}(x)$ its point-pair-distance density and geometric reduction factor. The measure, $a$, of $I$ has the expectation $E a=A_{\mathrm{w}}(1-\exp (-\Psi))$. Inserting $E a$ for $E a_{\mu}$ in Equation (17) and $p_{\mathrm{w}}(x), U_{\mathrm{w}}(x)$ and $U_{B}(x)$ for $p_{0}(x), U_{0}(x)$ and $U_{1}(x)$, and utilizing the relation

$$
(1-\exp (-\Psi)) A_{\mathrm{w}} \int p_{\mathrm{w}}(x) d x=(1-\exp (-\Psi)) A_{\mathrm{w}}=E a
$$

one obtains the variance, $\sigma_{l}^{2}$, of the measure of $I$ in $R^{n}$ :

$$
\begin{aligned}
\sigma_{l}^{2} & =A_{\mathrm{w}}^{2} \exp (-2 \Psi) \int_{0}^{z} p_{\mathrm{w}}(x)(\exp (\Psi U(x))-1) d x \\
& =A_{\mathrm{w}} \exp (-2 \Psi) \int_{0}^{z} w_{n} x^{n-1} U_{\mathrm{w}}(x)(\exp (\Psi U(x))-1) d x
\end{aligned}
$$

with $w_{1}=2, w_{2}=2 \pi, w_{3}=4 \pi$. The upper limit of the integration, $z$, is the largest point-pair distance of $W$ or, if smaller, the largest point-pair distance of the domains generating the Poisson process.

Pitts has treated a related problem of a Poisson process of diffuse entities with spherical symmetry. His result ([20], Equations (7), (8)) agrees with Equation 
(33) for the case of $n$-spheres and for the limit of a large domain $W$ (i.e. $\left.U_{\mathrm{w}}(z) \approx 1\right)$.

\section{Conclusions}

Formulae have been derived for the variance of the random intersection of two domains and for the variance of a Boolean scheme intersecting a sampling domain. The formulae contain the geometric reduction factors - or the closely related point-pair-distance distributions - of the domains.

Although this is not considered in the present paper, the results can be extended. One generalization is the inclusion of those cases where the intersecting regions are subdimensional. Another generalization is the admission of non-isotropic orientation of the intersecting domains. The geometric reduction factors depend then on distance and direction, and the integrations in Equations (17) and (33) run over both variables. Finally one can derive analogous formulae for the variances or the covariances of Minkowski functionals other than the $n$-dimensional measures. However, this last extension is restricted by the requirement that the measures - or, in the case of the covariances, one of the measures - need to be non-negative. Accordingly, it appears impossible to obtain comparable formulae for the variance of the Euler-Poincaré characteristic.

\section{Appendix}

The subsequent applications exemplify the relations for the variance of the intercept of two domains and for the variance of a Boolean scheme. The familiar lattice problem will be considered first, in order to indicate - without detailed treatment - the extension of Equation (17) to the case of subdimensional configurations.

Application of Equation (17) to the lattice problem. Consider the uniform and isotropic random placement of a domain, $F_{1}$, on a point grid. The variance, $\sigma^{2}$, of the number of covered grid points is to be determined.

Let $P(x)$ be the non-normalized distribution function of the point-pair distances of the $F_{0}$ :

$$
P(x)=A_{0} \int_{0}^{x} p_{0}(s) d s .
$$

$P(x)$ is the expected measure of $F_{0}$ at distances up to $x$ from a random point of $F_{0}$. With this function for $F_{0}$ and the geometric reduction factor $U(x)$ for the domain $F_{1}$, Equation (17) takes the form

$$
\sigma^{2}=\left(\int_{x=0}^{d} U(x) d P(x)-E_{\mu} a\right) E_{\mu} a
$$


The function $P(x)$ has the advantage that it applies also to a point grid. If each grid point is assigned unit measure, $P(x)$ is the number of grid points - or, depending on the type of grid, the mean number of grid points - within a circle of radius $x$ centred at a grid point, and $E_{\mu} a$ is the mean number of grid points covered by $F_{1}$. For a grid with one point per unit area $E_{\mu} a$ equals the measure, $A$, of $F_{1}$ (see, for example, [12]).

For a square grid of unit cell size in $R^{2}$ one has

(A.3) $P(x)=\sum_{i, j} 1, \quad$ (summation over all integers, $i, j$, with $i^{2}+j^{2} \leqq x^{2}$ ).

Accordingly one has the variance of the number of grid points covered:

$$
\sigma^{2}=\left(\sum_{i, j} U\left(\sqrt{i^{2}+j^{2}}\right)-A\right) A .
$$

The summation runs over all pairs $(i, j)$ of integers with $\sqrt{i^{2}+j^{2}}$ smaller than the maximum point-pair distance, $z$, of the figure. In numerical evaluations one enumerates only the combinations with $0 \leqq i \leqq j$, accounting for the omitted combinations by proper multiplicity factors. The resulting reduction of the number of terms is important for the analogous computations in $R^{3}$.

To apply Equation (A.4) to domains of standard geometry one uses the functions $U(x)$ which are listed in Equations (A.5)-(A.9). Figure 1 represents for circles of specified diameter, $d$, for squares of side length $d$, and for rectangles of side lengths $d$ and $\sqrt{2 d}$ the variance, $\sigma^{2}$, divided by the perimeter, $P$, of the figures. These and the analogous computations in $R^{3}$ were performed for different values $d$ in increments 0.02 . A square grid or a cubic grid of unit cell size has been assumed. The results for the circle are in agreement with the analytical treatment of Kendall [12] and Kendall and Rankin [13], and are also in general accord with the numerical data which Russell and Josephson [23] have given. The seeming irregularity of the dependence of $\sigma^{2}$ on $d$ is linked to the irregularity of the function $P(x)$. The broken lines in the graphs give the mean of $\sigma^{2} / P$ up to the specified value $d$. As averages of this mean over the interval $d=30$ to 40 the values $0.07288,0.07271$, and 0.07286 were obtained (values rounded to four digits). From the theory of Kendall for random ovals one derives the asymptotic value $\sigma^{2} / P=0.0728369$.

In the computations one utilizes the geometric reduction factor for the disc of diameter $d$, (see e.g. [14]):

$$
U(x)=\frac{2}{\pi}\left(\cos ^{-1}(X)-X\left(1-X^{2}\right)^{0.5}\right), \quad \text { for } 0 \leqq x \leqq d .
$$

Here and in formulae (A.7)-(A.9) the abbreviation $X=x / d$ is used.

The geometric reduction factor for a rectangle of side lengths $a$ and $b$ is 

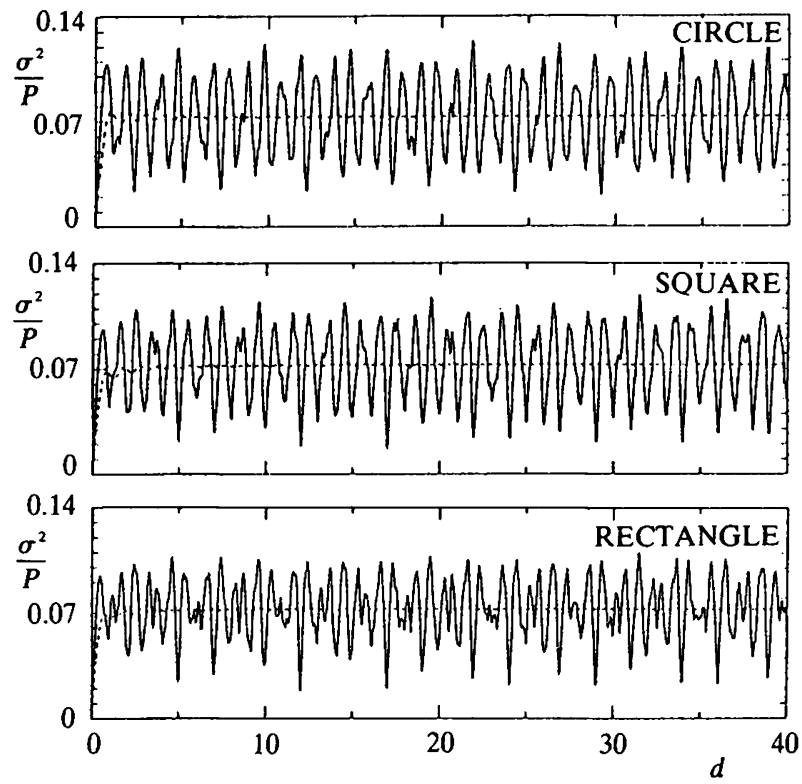

Figure 1 . The variance, divided by $P$, of the number of grid points covered by a randomly placed figure with random inclination. $P$ is the perimeter of the figure. $d$ is the diameter of the circle, the side length of the square, or the smaller side length of a rectangle with larger side length $\sqrt{2} \cdot d$. The square point grid has unit cell size.

Although the dependences on $d$ appear irregular, they are exact within the resolution of the graphs. The broken line gives the average of $\sigma^{2} / P$ up to the abscissa value

obtained by a method that has been described earlier [8]:

$U(x)=\frac{1}{\pi} \begin{cases}\pi-\frac{2(a+b) x}{a b}+\frac{x^{2}}{a b} & \text { for } 0<x<a \\ \frac{\pi}{2}+\sin ^{-1}\left(\frac{2 a^{2}}{x^{2}}-1\right)-\frac{a}{b}-2\left(\frac{x}{a}-\sqrt{\frac{x^{2}}{a^{2}}-1}\right) & \text { for } a<x<b \\ \sin ^{-1}\left(\frac{2 a^{2}}{x^{2}}-1\right)+\sin ^{-1}\left(\frac{2 b^{2}}{x^{2}}-1\right) & \\ +2\left(\sqrt{\frac{x^{2}}{b^{2}}-1}+\sqrt{\frac{x^{2}}{a^{2}}-1}\right)-\frac{a}{b}-\frac{b}{a}-\frac{x^{2}}{a b} & \text { for } b<x<\sqrt{a^{2}+b^{2}} .\end{cases}$

For the square of side length $d$ this reduces to:

(A.7) $U(x)=\frac{1}{\pi} \begin{cases}\pi-4 X+X^{2} & \text { for } 0 \leqq x \leqq d \\ 2 \sin ^{-1}\left(2 / X^{2}-1\right)+4\left(X^{2}-1\right)^{0.5}-2-X^{2} & \text { for } d \leqq x \leqq \sqrt{2} d .\end{cases}$ 
A cube of side length $d$ has the geometric reduction factor [19]:

$$
U(x)= \begin{cases}1-\frac{3 X}{2}+\frac{2 X^{2}}{\pi}-\frac{X^{3}}{4 \pi} & \text { for } 0 \leqq x \leqq d \\ \frac{6 \pi-1}{4 \pi X}-2+\frac{3 X}{2 \pi}+\frac{X^{3}}{2 \pi}+\frac{6 X}{\pi} \cos ^{-1}\left(\frac{1}{X}\right) & \text { for } d \leqq x \leqq \sqrt{2} d \\ -2\left(2 X^{2}+1\right)\left(X^{2}-1\right)^{0.5} & \text { for } \sqrt{2} d<x<\sqrt{3} d \\ \frac{6 \pi-5}{4 \pi X}+1-\frac{3(1+\pi) X}{2 \pi}-\frac{X^{3}}{4 \pi} & \text { 2(X } 2)\left(X^{2}-2\right)^{0.5}-\frac{6 A(X)}{\pi X}\end{cases}
$$

with $^{\dagger}$ :

$$
\left.A(X)=\operatorname{tg}^{-1}\left(\left(X^{2}-2\right)^{0.5}\right)+2+\operatorname{tg}^{-1}\left(X^{2}-1-X\left(X^{2}-2\right)^{0.5}\right)-X^{2} \operatorname{tg}^{-1}\left(X^{2}-2\right)^{-0.5}\right) .
$$

For the sphere of diameter $d$ one has the simple relation

$$
U(x)=1-\frac{3}{2} X+\frac{1}{2} X^{3} \text { for } 0 \leqq x \leqq d .
$$

The solutions for spheroids have been given elsewhere (prolate spheroid [6] and general case [10]).

Figure 2 gives for the cube of side length $d$, the sphere of diameter $d$, and for a prolate spheroid with the minor axes $d$ and larger axis $\sqrt{2} d$ the variance, $\sigma^{2}$, divided by the surface, $S$, of the bodies. For the cube and the sphere there are similar oscillations of the variance around its mean value as in the twodimensional case. For the spheroid - and the same has been found for oblate spheroids and prolate spheroids of other elongations - the amplitude of the oscillations decreases with increasing diameter. As in Figure 1, the broken lines give the mean values up to the specified values of $d$. As averages of this mean over the interval $d=30$ to 40 the values $0.06646,0.06680$, and 0.06684 were obtained for the cube, the sphere, and the prolate spheroid of elongation $\sqrt{2}$ (values rounded to four digits).

Numerical values for the Poisson process of domains. The subsequent examples serve to illustrate the magnitude of the variance of the Poisson process in typical cases. They also provide approximations applicable without precise evaluation of Equation (33).

Equation (33) can be rewritten in the modified form

$$
\sigma_{l}^{2}=A_{c} A_{w} \Psi \exp (-2 \Psi) C_{1} C_{2}
$$

- A typographical error in $A(X)$ in the original reference is here corrected. 

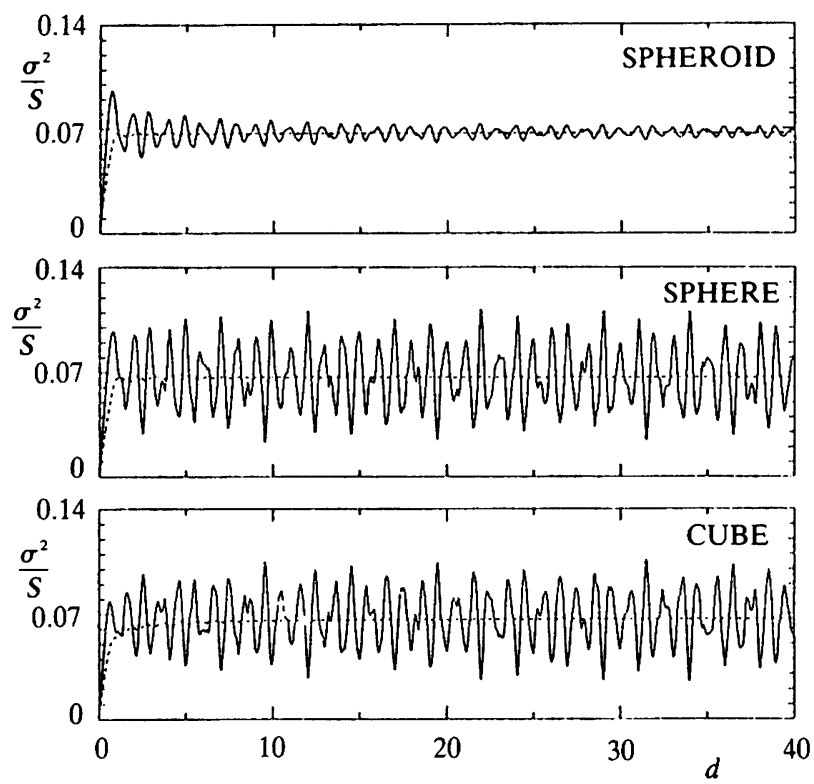

Figure 2 . The variance, divided by $S$, of the number of grid points covered by a randomly placed body of random inclination. $S$ is the surface of the body. $d$ is the side length of the cube, the diameter of the sphere, or the length of the two minor axes of the spheroid with major axis $\sqrt{2} \cdot d$. The cubic point grid has unit cell size.

The broken line gives the average of $\sigma^{2} / S$ up to the abscissa value

with

$$
C_{1}=\frac{1}{A_{c} \Psi} \int_{0}^{z}(\exp (\Psi U(x))-1) w_{n} x^{n-1} d x
$$

(A.12) $C_{2}=\int_{0}^{z}(\exp (\Psi U(x))-1) U_{\mathrm{w}}(x) w_{n} x^{n-1} d x / \int_{0}^{z}(\exp (\Psi U(x))-1) w_{n} x^{n-1} d x$.

$z$ is the maximum point-pair distance of the domains generating the Poisson process. $\boldsymbol{A}_{c}$ is the weighted average of the measure of the domains generating the Poisson process:

$$
A_{c}=\int A^{2}(p) \lambda(p) d p / \int A(p) \lambda(p) d p=\int_{0}^{z} U(x) w_{n} x^{n-1} d x .
$$

The last identity results from Equation (28) and the relation

$$
\int U(x, p) w_{n} x^{n-1} d x=A(p) .
$$

The specific form of Equation (A.10) is chosen because the terms $C_{1}$ and $C_{2}$ can be disregarded or can be treated as mere correction factors in certain instances. 


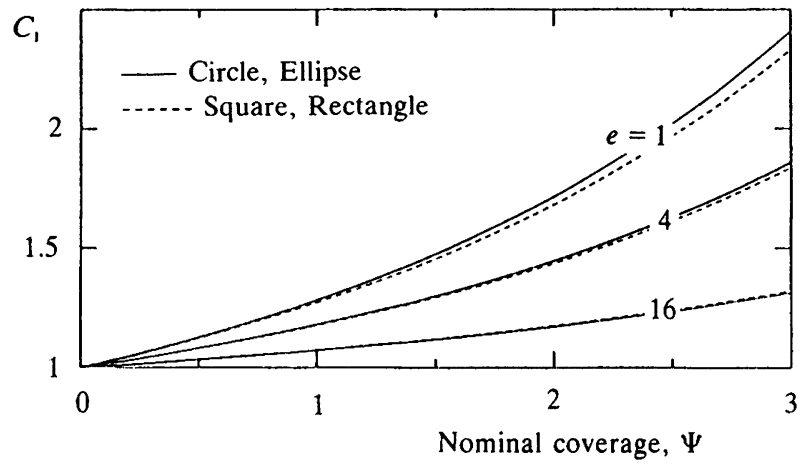

Figure 3. The value $C_{1}$ (see Equations (A.10) and (A.11)) for ellipses, and for rectangles of specified ratio, $e$, of the axes at different values of the nominal coverage

The term $C_{1}$ is always larger than unity. Figure 3 gives its value for ellipses and rectangles at different nominal coverages. The geometrical reduction factor for rectangles has been given in Equation (A.5); for ellipses it requires a numerical integration (see e.g. [10]).

As seen in Figure 3, $C_{1}$ is close to unity at moderate nominal coverages $(\Psi<1)$ which are common in applications. At specified $\Psi$ the largest values occur for the circle. An analogous statement applies for $R^{3}$, although the data, which are of less pragmatic importance, are not shown. Deviations from the circular or spherical form lead to smaller values of $C_{1}$, and reduced values of $C_{1}$ result also when the Boolean scheme is generated by circles or spheres of variable size. Although no proof is offered, one may surmise that the values for the $n$-sphere are generally upper bounds for $C_{1}$.

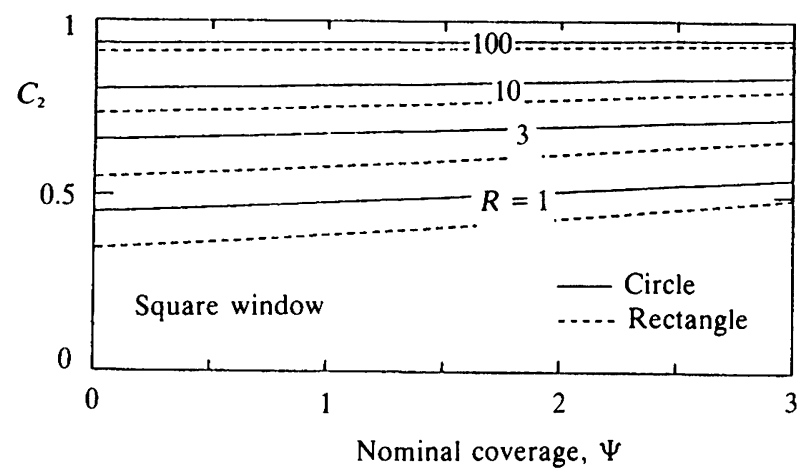

Figure 4. The value $C_{2}$ (see Equations (A.10) and (A.12)) for circles, and for rectangles with a ratio 4 of side lengths.

The curves result for a square window and for the specified ratio, $R$, of the area of the window to the area of the figure. The curves for a square are indistinguishable on this graph from those for the circle 
The approximation proposed by Pitts [20] corresponds to the relation $C_{1}=$ $(1-0.1149 \cdot \Psi)^{-2}$; this happens to be indistinguishable in Figure 3 from the curve for the square.

$C_{2}$ tends towards unity if - as has been generally assumed in earlier treatments - the sampling region, $W$, is large compared to the domains that generate the Poisson process $\left(U_{\mathrm{w}}(z) \approx 1\right)$. For a finite window $C_{2}$ is less than unity. Figure 4 gives numerical values for circles and for rectangles with ratio 4 of side lengths, and for a square window. The parameter $R$ is the ratio of the area of the window and the area of the figures generating the Poisson process.

\section{Acknowledgements}

I am grateful to my colleagues Dr Joachim Breckow (University of Würzburg) and Dr Marco Zaider (Columbia University) for discussions and helpful suggestions.

\section{References}

[1] Berger, M. J. (1970) Beta-ray dosimetry calculations with the use of point kernels. In Medical Radionuclides: Radiation Dose and Effects, ed. R. J. Cloutier et al., 63-86. (Also available as USAEC Report CONF-691212 from the National Technical Information Service, Springfield, VA 22161, USA).

[2] BLASCHKE, W. (1937) Integralgeometrie 21. Über Schiebungen. Math. Z. 42, 399-410.

[3] Bronowski, J. AND Neyman, J. (1944) The variance of the measure of a two-dimensional random set. Ann. Math. Statist. 16, 330-341.

[4] Coleman, R. (1979) An Introduction to Mathematical Stereology. Memoir Series, Dept of Theoretical Statistics, Institute of Mathematics, University of Aarhus.

[5] DAvY, P. J. (1976) Projected thick sections through multidimensional particle aggregates. $J$. Appl. Prob. 13, 714-722.

[6] Enns, E. G. And Ehlers, P. F. (1978) Random paths through a convex region. J. Appl. Prob. $15,144-152$.

[7] GARWOOD, F. (1947) The variance of the overlap of geometrical figures with reference to a bombing problem. Biometrika 14, 1-17. 264-276.

[8] Kellerer, A. M. (1981) Proximity functions for general right cylinders. Radiation Res. 86,

[9] Kellerer, A. M. (1983) On the number of clumps resulting from the overlap of randomly placed figures in a plane. J. Appl. Prob. 20, 126-135.

[10] Kellerer, A. M. (1984) Chord-length distributions and related quantities for spheroids. Radiation Res. 98, 425-437.

[11] KeLLeRER, H. G. (1984) Minkowski functionals of Poisson processes. Z. Wahrscheinlichkeitsth. 67, 63-84.

[12] Kendall, D. G. (1948) On the number of lattice points inside a random oval. Quart. J. Math. (Oxford) 19, 1-26.

[13] Kendall, D. G. and Rankin, R. A. (1953) On the number of points of a given lattice in a random hypersphere. Quart. J. Math. (2) 4, 178-189.

[14] Kendall, M. G. ANd Moran, P. A. P. (1963) Geometrical Probability. Griffin, London. $162-168$

[15] Kingman, J. F. C. (1965) Mean free paths in a convex reflecting region. J. Appl. Prob. 2,

[16] Matheron, G. (1967) Eléments pour une theorie des milieux poreux. Masson, Paris. 
[17] Matheron, G. (1974) Random Sets and Integral Geometry. Wiley, New York.

[18] MILES, R. E. (1979) Some new integral geometric formulae, with stochastic applications. $J$. Appl. Prob. 16, 592-606.

[19] PIEFKe, F. (1978) Beziehungen zwischen der Sehnenlängenverteilung und der Verteilung des Abstandes zweier zufälliger Punkte im Eikörper. Z. Wahrscheinlichkeitsth. 43, 129-134.

[20] PITTS, E. (1981) The overlap of random particles and similar problems: expressions for variance of coverage and its analogue. SIAM J. Appl. Math. 41, 493-498.

[21] Robbins, H. E. (1944) On the measure of a random set. Ann. Math. Statist. 15, 70-74.

[22] Robiins, H. E. (1945) On the measure of a random set, II. Ann. Math. Statist. 16, 342-347.

[23] Russell, A. M. AND Josephson, N. S. (1965) Measurement of area by counting. J. Appl. Prob. 2, 339-351.

[24] Santaló, L. (1947) On the first two moments of the measure of a random set. Ann. Math. Statist. 18, 37-49.

[25] Santalo, L. (1953) Introduction to Integral Geometry. Herman, Paris.

[26] WeIL, W. (1984) Densities of Quermassintegrals for stationary random sets. In Stochastic Geometry, Geometric Statistics, Stereology ed. R. Ambartzumian and W. Weil, Teubner Texte zur Mathematik 35, Teubner, Leipzig, 233-247. 